\title{
DIABETES MELLITUS AND BONE HEALTH
}

\author{
Kumar Gaurav \\ Department of Biotechnology \\ Jain University, Bangalore, Karnataka, India
}

\begin{abstract}
Diabetes is said to be derived from a Greek word Diabetes which means siphon. Siphon means to pass through and the Latin word Mellitus meaning sweet. Diabetes Mellitus is a disease which disrupts normal metabolism by the process of elevation in blood glucose levels. Insulin hormone cannot move glucose into the cells from the blood which results in increased accumulation of glucose in blood. As of now there is no cure for diabetes but with regular exercise and proper meal planning, one can control the diabetes. Diabetes comes in different forms such as Prediabetes, T1DM, T2DM and Gestational diabetes. A diabetic person with long history of diabetes is prone to impaired bone structure and has high risks of bone fracture. So, the controlling of diabetes become necessary to avoid complications regarding bone fragility. In this review, I will emphasis on the impact of diabetes mellitus on bones.
\end{abstract}

Keywords- Diabetes Mellitus, Insulin, Pancreas, Hyperglycemia, Fracture

\section{INTRODUCTION}

Diabetes mellitus have caused decrease in the quality of the bone and have hiked risks of fractures of bones in patients. A study which involved 783213 participants showed that diabetic people are more prone to bone fractures when compared to control group and it also showed that patients affected with T1DM were more susceptible to hip fractures as compared to patients affected with T2DM while the relative risk of T2DM was 1.34-1.7 and that of T2DM was 5.76-6.3 $3^{1,2}$. The people who are affected with diabetes and are going through a fracture recovery stays longer in hospital but they generally recover $^{3}$. The most important causes behind increased risk of fracture are decreased bone strength, poor bone microarchitecture and increased risk of fall in elderly people. ${ }^{4}$ Low bone mineral density is obvious during the time of diagnosis ${ }^{5}$. Vestergaard et al. (2009) concluded that diabetes, whether T1DM or T2DM carry an increased risk of fracture ${ }^{5,6}$ but again T2DM has a high risk of fracture among both the types due to differences in bone quality between T1DM and $\mathrm{T}_{2} \mathrm{DM}^{6}$. Increase of glucose levels in blood are said to be related with an increasing loss of calcium in urine, ${ }^{7}$ so, one can say that increased levels of glucose in blood may have harmful effect on bones ${ }^{8}$. The motto of this review is to outline the basics of diabetes and its types, along with the pathogenesis and prevention of the disease. To achieve this aim, various number of articles related to diabetes and fracture risk were evaluated using PubMed Central and different diabetes related journals. No year restriction was followed. Snowballing technique was also performed to find the best literature articles.

\section{Diabetes Mellitus AND Bones}

Type 1 diabetes mellitus is a disease which results due to insulin deficiency and increased blood glucose level due to autoimmune destruction of pancreatic $\beta$-cells ${ }^{9}$. T1DM increases the risk of hip fractures ${ }^{10}$. It is also associated with some of the major complications such as retinopathy, nephropathy, neuropathy, cardiovascular diseases ${ }^{9}$. Type 2 Diabetes Mellitus is defined by insulin resistance with increased amount of insulin i.e. hyperinsulinemia and impair insulin secretion ${ }^{11}$ which is also associated with the increased risk of fractures. The increasing number of fractures is more likely to increase the cost associated with these fractures, hence increasing the economic burden of bone fracture to society ${ }^{12}$. The risk of fall increases in the people suffering from DM but still falls are not completely related with the increased fracture risk ${ }^{13}$. Therefore, changes such as metabolic or biochemical which are associated with DM might have altered the bone microarchitecture and tissues ${ }^{14}$. Various other factors such as high sugar in blood, decreased concentration of insulin and autoimmune inflammation, vitamin $\mathrm{D}$, reduced levels of IGF-1 might have increased the chances of bone fragility $^{14,15}$. Peripheral vascular diseases may also cause bone fragility $^{5}$. These factors also play a crucial role in impairing osteoblast differentiation ${ }^{15}$. T1DM is correlated with the small reduction of bone mineral density and increased chances of fractures with the Hip Z score of $-0.37 \pm 0.16$ and T2DM patients have hip $\mathrm{z}$ score of $0.27 \pm 0.16$ with an increase in fracture risk ${ }^{16}$. This raises the question that if increased bone mineral density is protective against fractures. The complete absence of insulin in T1DM along with the combination of low levels of IGF-1 decreases the rate of bone formation by applying an inhibitory effect on osteoblasts during the early stages of disease ${ }^{17}$. As we know that T1DM generally occurs in children and young adults, the complete absence of insulin corresponds with a stage of skeletal maturation, which affects the bone accumulation and development ${ }^{11,16}$. High levels of blood glucose generates a high concentration of advanced glycation end products due to which bone strength is reduced $^{18,19}$. Bone remodeling and bone loss could be accelerated with an hike in inflammation and its associated 
cytokine $^{19,20,21}$. During the study of rat models of T1DM, Verhaeghe $\mathrm{J}$ et al. reported that bone histology and bone markers indicated reduced osteoblast activity combined with normal or reduced osteoclast activity ${ }^{22}$. Some of the studies related to bone turnover in T1DM have reported increased resorption in humans ${ }^{19}$. In 1980, Hunter Heath and his colleagues in their study using mayo clinic records reported that increased risk of fracture was not associated with diabetes. They also concluded that T2DM is associated with increased weight which provides protection from most fractures $^{23}$. Although increased risk of hip fractures has been reported in various recent cohort studies ${ }^{19}$. Some of the studies have reported that sites such as proximal humerus, foot and ankle has high fracture risk due to diabetes ${ }^{24,25}$. Also, one cohort study reported a decreased fracture risk in old age women suffering from diabetes, considering all non-vertebral fracture sites combined ${ }^{19,26}$. Kemink S.A. et al. in their study reported that men suffering from T1DM are more prone to osteopenia or osteoporosis. They also reported that there was a negative correlation between duration of T1DM with bone mineral density only in men ${ }^{11,27}$. Some of the factors associated with low bone mass in T1DM are use of estrogen/gestagen based oral contraceptives in women, smoking in both men and women which may be responsible for increased risk of fractures ${ }^{28,29}$. Some of the studies which observed determinant conditions for falls and injurious falls have not made clearer about the relation between diabetes and fractures ${ }^{19}$. After statistically adjusting for various factors such as physical inactivity, impaired vision, impaired motor abilities related to fall risk, Forsen et al. in their study by working on the data from Nord-Trondelag Health Survey reported an increased risk of hip fractures in men and women above 50 years of age ${ }^{30}$. In a similar way, even after adjusting for various fall related factors, Ottenbacher et al. in their study of old age Mexican-American people found an 50\% increased risk of hip fracture ${ }^{19,31}$. This clearly suggests that falls or injuries are not solely responsible for fracture incidence in diabetes. Decreased bone strength may be the reason behind the increased fracture incidence in diabetic patients.

T1DM is mostly reported in children, adolescents and young adults. So, the insulinopenia coexist with skeletal immaturity which affects bone growth ${ }^{11}$. So, it has led to a hypothesis that insulin assists as an anabolic factor for bones. Also, the hypothesis that poor quality of diabetic bone is not associated with the lower density is supported by the rodent models ${ }^{19}$. Various types of studies on spontaneously diabetic rats and streptozotocin-induced diabetes have reported decreased strength of bones ${ }^{22,32,33}$. Decreased bone strength in diabetes can be explained by the accumulation of advanced glycation end products in collagen of bones. AGEs are said to be reduce elasticity and increase the permeability of blood vessels ${ }^{34}$. Wang et al. in their study on human cadaveric bone reported that a high concentration of AGE was associated with a decreased strength ${ }^{35}$.
Another reason for the decreased bone strength in older diabetic people is rapid loss of bones. Krakauer et al. on his study of 19 people with T2DM found no loss of bone mineral density $^{36}$. In various studies, great weight loss has been reported to be correlated with bone loss ${ }^{37,38}$.

\section{DIABETES MANAGEMENT AND PREVENTION}

With a change in living style and treatment methods, a diabetic person can achieve good metabolic control over the diabetes ${ }^{39}$. Maintaining a good diet plan can help diabetic people achieve the normal blood glucose, blood pressure, weight and also lipid profile ${ }^{40}$. Various studies have also reported that $\mathrm{HbA} 1 \mathrm{c}$ can be reduced through proper nutritional recommendation ${ }^{41,42}$. Consumption of alcohol in moderate quantity will not harm the blood sugar levels but can improve the same and reduce cardiovascular events ${ }^{39}$. Omega-3 rich product consumption can also be helpful in preventing cardiovascular disease ${ }^{43}$. Physical activity and exercises are the most helpful tool in treatment of diabetes.

Various kinds of oral agents are also helpful in treatment of diabetes. Metformin is one of the best oral agents used by diabetic patients ${ }^{44,45}$. Intake of metformin activates AMPactivated protein kinase and it also decreases lipopolysaccharide levels ${ }^{46}$. Metformin inhibits synthesis of glucose by different mechanisms such as ${ }^{47}$ :

a) by activating hepatic $\mathrm{AMPK}^{48}$.

b) by inhibiting production of glucagon induced CAMP production $^{49}$.

c) by activating AMPK through increased ATP/AMP ratio in the mitochondrial electron transport chain ${ }^{50}$.

d) By inhibiting mitochondrial glycerol phosphate dehydrogenase ${ }^{51}$.

Sulfonylureas and glinides are another oral agent used by the diabetic people ${ }^{39}$. They increase insulin secretion by regulating ATP-sensitive potassium channels ${ }^{52}$. Various other oral agents are available such as acarbose, miglitol and voglibose ${ }^{53}$ which are alpha-glucosidase enzyme located in the border membrane of the small intestine ${ }^{54}$. Dapagliflozin, canagliflozin and empagliflozin are three oral antidiabetic drugs which represents sodium glucose co-transporter- 2 inhibitor and are of great use. $^{39}$

\section{CONCLUSION}

Diabetes Mellitus is becoming a matter of concern in $21^{\text {st }}$ century. More and more people are being affected by the diabetes worldwide. With the two types of diabetes (T1DM and T2DM) one should exercise proper caution in their lifestyle. Increase in blood sugars are a matter of concern and early diagnosis of the same can reduce mental and physical burden of the patient. T1DM is mainly diagnosed in the young adults and children which leads to insulinopenia and T2DM is diagnosed into the old people over a course of time. With the increase in risk fractures in both of the types, more and more 


\section{International Journal of Engineering Applied Sciences and Technology, 2021 \\ Vol. 6, Issue 2, ISSN No. 2455-2143, Pages 240-244 \\ Published Online June 2021 in IJEAST (http://www.ijeast.com)}

cases are being reported about the bone fractures which also increases an economy burden on the country. Clinical management of the diabetes is possible through the wide range of injectable or oral agents available in the market. Future research should study about the anti-diabetic drugs with no side effects and gene therapy for the complete treatment of diabetes.

\section{REFERENCE}

1. Janghorbani M, Van Dam RM, Willett WC, Hu FB. Meta-Analysis Systematic Review of Type 1 and Type 2 Diabetes Mellitus and Risk of Fracture. doi:10.1093/aje/kwm106

2. Fan Y, Wei F, Lang Y, Liu Y. Diabetes mellitus and risk of hip fractures: a meta-analysis. Osteoporos Int. 2016;27(1):219-228. doi:10.1007/s00198-015-3279-7

3. Norris R, Parker M. Diabetes mellitus and hip fracture: A study of 5966 cases. Injury. 2011;42(11):1313-1316. doi:10.1016/j.injury.2011.03.021

4. Oei L, Zillikens MC, Dehghan A, et al. High bone mineral density and fracture risk in type 2 diabetes as skeletal complications of inadequate glucose control: The Rotterdam study. Diabetes Care. 2013;36(6):1619-1628. doi:10.2337/dc12-1188

5. Al-Hariri M. Sweet Bones: The Pathogenesis of Bone Alteration in Diabetes. J Diabetes Res. 2016;2016:15. doi:10.1155/2016/6969040

6. Vestergaard P, Rejnmark L, Mosekilde L. Diabetes and its complications and their relationship with risk of fractures in type 1 and 2 diabetes. Calcif Tissue Int. 2009;84(1):45-55. doi:10.1007/s00223-0089195-5

7. McNair P, Madsbad S, Christensen MS, et al. Bone mineral loss in insulin-treated diabetes mellitus: Studies on pathogenesis. Acta Endocrinol (Copenh). 1979;90(3):463-472. doi:10.1530/acta.0.0900463

8. Gopalakrishnan V, Vignesh RC, Arunakaran J, Aruldhas MM, Srinivasan N. Effects of glucose and its modulation by insulin and estradiol on BMSC differentiation into osteoblastic lineages. Biochem Cell Biol. 2006;84(1):93-101. doi:10.1139/o05-163

9. Wang JF, Lee MS, Tsai TL, et al. Bone Morphogenetic Protein-6 Attenuates Type 1 Diabetes Mellitus-Associated Bone Loss. Stem Cells Transl Med. 2019;8(6):522-534. doi:10.1002/sctm.18-0150

10. Nicodemus KK, Folsom AR. Type 1 and type 2 diabetes and incident hip fractures in postmenopausal women. Diabetes Care. 2001;24(7):1192-1197. doi:10.2337/diacare.24.7.1192
11. LC H, CC B, SK S, H D. Osteoporosis in patients with diabetes mellitus. J Bone Miner Res. 2007;22(9):1317-1328. doi:10.1359/JBMR.070510

12. Nyman JS, Even JL, Jo CH, et al. Increasing duration of type 1 diabetes perturbs the strength-structure relationship and increases brittleness of bone. Bone. 2011;48(4):733-740. doi:10.1016/j.bone.2010.12.016

13. Starup-Linde J, Frost M, Vestergaard P, Abrahamsen B. Epidemiology of Fractures in Diabetes. Calcif Tissue Int. 2017;100(2):109-121. doi:10.1007/s00223-016-0175-X

14. Lekkala S, Taylor EA, Hunt HB, Donnelly E. Effects of Diabetes on Bone Material Properties. Curr Osteoporos Rep. 2019;17(6):455-464. doi:10.1007/s11914-019-00538-6

15. Zhukouskaya V V., Eller-Vainicher C, Shepelkevich AP, Dydyshko Y, Cairoli E, Chiodini I. Bone health in type 1 diabetes: Focus on evaluation and treatment in clinical practice. $\mathrm{J}$ Endocrinol Invest. 2015;38(9):941-950. doi:10.1007/s40618-015-0284-9

16. Murray CE, Coleman CM. Impact of diabetes mellitus on bone health. Int J Mol Sci. 2019;20(19). doi:10.3390/ijms20194873

17. Napoli N, Chandran M, Pierroz DD, Abrahamsen B, Schwartz A V., Ferrari SL. Mechanisms of diabetes mellitus-induced bone fragility. Nat Rev Endocrinol $2016 \quad 134.2016 ; 13(4): 208-219$. doi:10.1038/nrendo.2016.153

18. RG P, AJ B. Glycation of collagen: the basis of its central role in the late complications of ageing and diabetes. Int J Biochem Cell Biol. 1996;28(12):12971310. doi:10.1016/S1357-2725(96)00079-9

19. Schwartz A V. Diabetes Mellitus: Does it Affect Bone? Calcif Tissue Int. 2003;73(6):515-519. doi:10.1007/S00223-003-0023-7

20. JC P, MA C. Is type II diabetes mellitus a disease of the innate immune system? Diabetologia. 1998;41(10):1241-1248. doi: $10.1007 / \mathrm{S} 001250051058$

21. SC M, RL J. Bone marrow, cytokines, and bone remodeling. Emerging insights into the pathophysiology of osteoporosis. $\mathrm{N}$ Engl J Med. 1995;332(5):305-311. doi:10.1056/NEJM199502023320506

22. J V, AM S, TA E, et al. Brittle bones in spontaneously diabetic female rats cannot be predicted by bone mineral measurements: studies in diabetic and ovariectomized rats. J Bone Miner Res. 1994;9(10):1657-1667. doi:10.1002/JBMR.5650091021

23. Heath HI, Melton LJI, Chu C-P. Diabetes Mellitus and Risk of Skeletal Fracture. 


\section{International Journal of Engineering Applied Sciences and Technology, 2021 \\ Vol. 6, Issue 2, ISSN No. 2455-2143, Pages 240-244 \\ Published Online June 2021 in IJEAST (http://www.ijeast.com)}

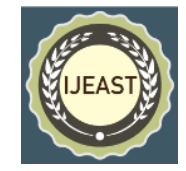

http://dx.doi.org/101056/NEJM198009043031008.

2009;303(10):567-570.

doi:10.1056/NEJM198009043031008

24. AV S, DE S, KE E, et al. Older women with diabetes have an increased risk of fracture: a prospective study. J Clin Endocrinol Metab. 2001;86(1):32-38. doi:10.1210/JCEM.86.1.7139

25. KK N, AR F. Type 1 and type 2 diabetes and incident hip fractures in postmenopausal women. Diabetes Care. doi:10.2337/DIACARE.24.7.1192

2001;24(7):1192-1197.

26. PL van D, RP S, H B, et al. Bone density in noninsulin-dependent diabetes mellitus. The Rotterdam Study. Ann Intern Med. 1995;122(6):409-414. doi:10.7326/0003-4819-122-6-199503150-00002

27. SA K, AR H, LM S, JA L, AG S. Osteopenia in insulin-dependent diabetes mellitus; prevalence and aspects of pathophysiology. J Endocrinol Invest. 2000;23(5):295-303. doi:10.1007/BF03343726

28. M M-T, E J, F E-J, PJ L-I, JD L. Bone mineral density measured by dual X-ray absorptiometry in Spanish patients with insulin-dependent diabetes mellitus. Calcif Tissue Int. 1996;58(5):316-319. doi:10.1007/BF02509378

29. H L, CM F, T C, et al. A population-based study of bone mineral density in women with longstanding type 1 (insulin dependent) diabetes. Diabetes Res Clin Pract. 1998;40(1):31-38. doi:10.1016/S01688227(98)00012-6

30. L F, HE M, K M, TH E. Diabetes mellitus and the incidence of hip fracture: results from the NordTrøndelag Health Survey. Diabetologia. 1999;42(8):920-925. doi:10.1007/S001250051248

31. KJ O, GV O, MK P, JS G, KS M. Diabetes mellitus as a risk factor for hip fracture in mexican american older adults. J Gerontol A Biol Sci Med Sci. 2002;57(10). doi:10.1093/GERONA/57.10.M648

32. GK R, L S-B, S H, CS E. The biomechanical integrity of bone in experimental diabetes. Diabetes Res Clin Pract. 2001;54(1):1-8. doi:10.1016/S01688227(01)00273-X

33. J V, E van $\mathrm{H}$, WJ $\mathrm{V}$, et al. Bone and mineral metabolism in BB rats with long-term diabetes. Decreased bone turnover and osteoporosis. Diabetes. 1990;39(4):477-482. doi:10.2337/DIAB.39.4.477

34. M B, A C, H V. Advanced glycosylation end products in tissue and the biochemical basis of diabetic complications. $\mathrm{N}$ Engl J Med. 1988;318(20):1315-1321. doi:10.1056/NEJM198805193182007
35. X W, X S, X L, CM A. Age-related changes in the collagen network and toughness of bone. Bone. 2002;31(1):1-7. doi:10.1016/S8756-3282(01)00697-4

36. JC K, MJ M, NF B, DS R, FW W, AM P. Bone loss and bone turnover in diabetes. Diabetes. 1995;44(7):775-782. doi:10.2337/DIAB.44.7.775

37. Black SA, Ray LA, Markides KS. The prevalence and health burden of self-reported diabetes in older Mexican Americans: Findings from the Hispanic Established Populations for Epidemiologic Studies of the Elderly. Am J Public Health. 1999;89(4):546-552. doi:10.2105/AJPH.89.4.546

38. HC L, WC K, RL H. Changes in BMI and weight before and after the development of type 2 diabetes. Diabetes Care. 2001;24(11):1917-1922. doi:10.2337/DIACARE.24.11.1917

39. Marín-Peñalver JJ, Martín-Timón I, SevillanoCollantes C, Cañizo-Gómez FJ del. Update on the treatment of type 2 diabetes mellitus. World J Diabetes.

2016;7(17):354 doi:10.4239/WJD.V7.I17.354

40. K E, MI M, M C, et al. Effects of a Mediterraneanstyle diet on the need for antihyperglycemic drug therapy in patients with newly diagnosed type 2 diabetes: a randomized trial. Ann Intern Med. 2009;151(5):306-314. doi:10.7326/0003-4819-151-5200909010-00004

41. MJ F, JL B, J G-P, MA P. Evidence-based nutrition practice guidelines for diabetes and scope and standards of practice. J Am Diet Assoc. 2008;108(4 Suppl 1). doi:10.1016/J.JADA.2008.01.021

42. RC A, AR C, AA M, et al. Diet or diet plus physical activity versus usual care in patients with newly diagnosed type 2 diabetes: the Early ACTID randomised controlled trial. Lancet (London, England). 2011;378(9786):129-139. doi:10.1016/S0140-6736(11)60442-X

43. BE K, AE J, L B, LG B, BO V. Fatty fish in the diet of patients with type 2 diabetes: comparison of the metabolic effects of foods rich in n-3 and n-6 fatty acids. Am J Clin Nutr. 2011;94(1):26-33. doi:10.3945/AJCN.110.006221

44. Inzucchi SE, Bergenstal RM, Buse JB, et al. Management of Hyperglycemia in Type 2 Diabetes: A Patient-Centered Approach. Diabetes Care. 2012;35(6):1364-1379. doi:10.2337/DC12-0413

45. Nathan DM, Buse JB, Davidson MB, et al. Medical Management of Hyperglycemia in Type 2 Diabetes: A Consensus Algorithm for the Initiation and Adjustment of Therapy. Diabetes Care. 2009;32(1):193-203. doi:10.2337/DC08-9025 
46. G Z, R M, Y L, et al. Role of AMP-activated protein kinase in mechanism of metformin action. $\mathrm{J}$ Clin Invest. 2001;108(8):1167-1174. doi:10.1172/JCI13505

47. R S. Mechanism of Metformin: A Tale of Two Sites. Diabetes Care. 2016;39(2):187-189. doi:10.2337/DCI15-0013

48. AJ G, MJ A, JI B, et al. CONSENSUS STATEMENT BY THE AMERICAN ASSOCIATION OF CLINICAL ENDOCRINOLOGISTS AND AMERICAN COLLEGE OF ENDOCRINOLOGY ON THE COMPREHENSIVE TYPE 2 DIABETES MANAGEMENT ALGORITHM--2016 EXECUTIVE SUMMARY. Endocr Pract. 2016;22(1):84-113. doi:10.4158/EP151126.CS

49. FP C, D C, L D, P S, MA M. Sleep duration predicts cardiovascular outcomes: a systematic review and meta-analysis of prospective studies. Eur Heart J. 2011;32(12):1484-1492. doi:10.1093/EURHEARTJ/EHR007

50. J M, É D, JP C. Inadequate sleep as a contributor to obesity and type 2 diabetes. Can $\mathrm{J}$ diabetes. 2013;37(2):103-108. doi:10.1016/J.JCJD.2013.02.060

51. H A, L H. Current understanding of metformin effect on the control of hyperglycemia in diabetes. J Endocrinol. 2016;228(3):R97-R106. doi:10.1530/JOE-15-0447

52. J B, A C, WH V-C, AP B, L A-B. Insulin secretagogues, sulfonylurea receptors and K(ATP) channels. Curr Pharm Des. 2005;11(21):2699-2716. doi: $10.2174 / 1381612054546879$

53. TP W, M N, PM C, X C, JB K, GD B. A high-protein diet with resistance exercise training improves weight loss and body composition in overweight and obese patients with type 2 diabetes. Diabetes Care. 2010;33(5):969-976. doi:10.2337/DC09-1974

54. M A, K O, M S. Antidiabetic agents in patients with chronic kidney disease and end-stage renal disease on dialysis: metabolism and clinical practice. Curr Drug Metab. 2011;12(1):57-69. doi:10.2174/138920011794520053 